\title{
EFEITO DA VELOCIDADE DE OPERAÇÃO DO ARADO DE DISCOS, DE ARRASTO, NA COMPONENTE LONGITUDINAL DO ESFORÇO TRATÔRIO, EM SOLO DE CLASSE TEXTURAL AREIA *
}

\author{
Antônio de Pádua Sousa ** \\ João B. Guimarães Jr. ** \\ ODILON SAAD ***
}

\section{RESUMO}

O presente trabalho fol realizado com o propósito de estudar o efeito do aumento de velocidade sobre os valores da componente longitudinal do esforço tratório. Foi usado um arado de discos, de arrasto. Para a medição da componente longitudinal do esforço tratório fol utilizado um dispositivo de acoplamento na barra do trator, onde fol inserido um dinamômetro eletromecânico PIAB.

Os ensaios foram realizados, em condições de campo, em um solo de classe textural areia. As velocidades de deslocamento foram em número de $4: 2,86 \mathrm{~km} / \mathrm{h}, 3,61 \mathrm{~km} / \mathrm{h}, 4,74 \mathrm{~km} / \mathrm{h}$ e $6,64 \mathrm{~km} / \mathrm{h}$.

Nas condições em que foram realizados os ensaios, determinou-se a seguinte equação:

$\mathrm{K}=2,64 \mathrm{v}+62,88$, sendo $\mathrm{K}=$ força especifica média em $\mathrm{kgf} / \mathrm{dm}^{2}$ $\checkmark=$ velocidade de deslocamento em $\mathrm{km} / \mathrm{h}$.

\section{INTRODUÇAOO}

No que tange às pesquisas a respeito da aração, envolvendo o sistema solo-máquina, é de se ver que uma análise qualitativa de tal sistema implica na consideração de diversos aspectos. Assim é que, atualmente, tendo-se em mira o aumento do rendimento em área trabalhada,

- Trabalho apresentado na XXVI Reunião Anual da S. B. P. C. - 10 a 17 de julho de 1974 - Recife, Pernambuco. Entregue para publicação em 5/11/1974.

* Respectivamente, Professor Assistente e Professor Titular, do Departamento de Engenharia Rural da F.C.M.B.B. - Botucatu - S.P.

*** Professor Adjunto do Departamento de Engenharia Rural da E. S. A. L. Q. - U. S. P. Piracicaba - SP. 
há uma tendência manifesta no sentido de uma especial preocupação com o aumento da velocidade de operação. Este assunto foi objeto da Assembléia Anual realizada em 1968, nos Estados Unidos pela Sociedade de Engenheiros Automobilistas, na qual se apresentou um informe de engenheiros pesquisadores da Deere \& Company. Em 1971, essa mesma Sociedade dedicou uma seção completa aos tratores e implementos de alta velocidade.

O aumento da velocidade de operação tem implicação sobre o sistema solo-máquina, em razão da ação da energia cinética aplicada sobre as características físicas do solo e suas possíveis consequências para as culturas a serem instaladas e nos elementos orgânicos da máquina.

Com relação ao arado, como u'a máquina cujo projeto deve ser aperfeiçoado em função das condições operacionais, tem particular interesse o conhecimento das forças ativas e reativas a que está submetido em diversas velocidades de operação. evidente também que o seu desempenho, no que se refere ao tempo e a energia aplicada, requer investigações mais profundas em nosso país, principalmente no estabelecimento de condições limites para os arados convencionais e na orientação de novos projetos, tendo em vista o sistema solo-máquina.

0 presente trabalho tem por objetivo investigar a influência da variação da velocidade de operação em arado de discos, de arrasto, na componente longitudinal do esforço tratório, com vistas a fornecer subsídios a usuários com respeito a requerimentos de potência e a projetistas para o aprimoramento de projetos.

\section{REVISAO DA LITERATURA}

As informações concernentes ao efeito da variação da velocidade de deslocamento do arado de disco, sobre o valor da componente longitudinal do esforço tratório existentes na literatura são relativamente escassas. Dos diversos trabalhos compulsados na literatura técnica, evidenciaram-se os seguintes:

McKIBBEN e REED (1952) afirmaram ser evidente o aumento da componente longitudinal do esforço tratório quando é aumentada a velocidade. Computaram dados de um grande número de ensaios com implementos agrícolas de preparo inicial do solo. Os dados foram explicitados em porcentagem de «tração» para um determinado implemento operando a 3 milhas/h. Foram estabelecidas curvas por meios não analíticos. As curvas indicaram que o percentual de aumento na componente longitudinal de um implemento quando a velocidade é aumentada acima de 3 milhas/h, será entre 5 a 15 vezes o valor correspondente a exponencial 1,5 do acréscimo de velocidade.

PAYNE (1956) utilizou-se da mecânica de solos para estudar ou predizer a componente longitudinal do esforço tratório e o volume de solo removido pelos implementos agrícolas. Pelas determinações da resistên- 
cia ao cizalhamento do solo e da resistência ao deslizamento solo-implemento, e do peso específico aparente do solo foi capaz de predizer com alguma segurança as forças que atuam no dente retangular e vertical empregado. Encontrou um aumento na componente longitudinal do esforço tratório.

GETZLAFF e SOHNE (1959), em ensaios de campo com o objetivo de determinar as forças e exigências de potência em arado de discos girando livremente e movidos por unidade motriz auxiliar, utilizaram dois tipos de disco, um dentado e um liso, e dois níveis de velocidade, 1,0 e $1,9 \mathrm{~m} / \mathrm{s}$. Do ponto de vista da velocidade de operação, concluiram que a velocidade de aração teve pouca influência na inclinação das curvas estabelecidas, alterando ligeiramente os valores absolutos.

JOHNSTON e BIRTWISTLE (1963), em experimentos de campo, utilizaram-se de um arado de arrasto com dois discos, especialmente construído. Estudaram diferentes combinações de ângulos, diâmetros, concavidade e bordos dos discos, largura, profundidade e velocidade de aração. Os resultados obtidos foram analisados estatisticamente e evidenciaram que nos três tipos de solos utilizados, esta última, teve um efeito altamente significativo na componente longitudinal do esforço tratório.

MIALHE e SILVEIRA (1971) fizeram ensaios de campo, empregando arado de discos, de arrasto, deslocando-se com 3 diferentes velocidades: 3,$86 ; 5,14$ e $6,20 \mathrm{~km} / \mathrm{h}$, em solo caracterizado como sendo da Série Sertãozinho. Os autores concluiram principalmente, que o aumento de velocidade de deslocamento do arado acarreta um aumento da potência para tracioná-lo, sugerindo que isto seja provocado mais pelo aumento da velocidade em si do que pela variação do coeficiente de resistência do solo.

\section{MATERIAIS E MÉTODOS}

\section{Materiais}

Os materiais utilizados no presente trabalho abrangem, o trator, 0 arado, o dinamógrafo e 0 solo.

Trator: - para tracionar o arado, foi utilizado um trator de rodas pneumáticas, marca Massey-Ferguson, modelo MF-178.

Arado: - empregou-se um arado de 3 discos, de arrasto, marca Civemasa, modelo ARC-1.000, equipado com 3 discos de $710 \mathrm{~mm}$ de diâmetro, pesando 1.300 quilos. Os ângulos de ataque foram $42^{\circ}$ - para o ângulo horizontal e $20^{\circ}$ para o ângulo vertical.

Dinamógrafo: - para medição e registro dos valores da componente longitudinal foi utilizado um dinamógrafo, composto de um transdutor eletromecânico da marca PIAB com capacidade para 1.000 quilos. Tendo-se em vista o propósito da medição e considerando-se a capacidade máxima do dinamômetro, foi necessário adotar-se um dispositivo na barra de tração, 
afim de se atenuarem as deformações percebidas pelo transdutor numa gama aceitável pelo sistema. Esse dispositivo se baseia na decomposição do esforço tratório em duas componentes. Sendo adotado no projeto a atenuação de 3:1. A unidade de registro consiste num medidor potenciométrico tipo pena, marca NORMA, com sensibilidade de $20 \mathrm{kgf}$ e acuracidade de $\pm 0,6 \%$ da capacidade máxima (FIGURAS 1 e 2 ).

Solo: - os ensaios foram desenvolvidos em solo cuja análise granulométrica é apresentada no QUADRO 1, e ocorre na Estação Experimental de Botucatu, numa situação de relevo normal ondulado. 0 declive de $7,5 \%$ apresenta uma forma plana. Por ocasião do desenvolvimento desse ensaio não havia cobertura vegetal na superfície do solo, face a uma recente queimada.

QUADRO 1 - Características físicas do solo de classe textural areia, à profundidade de $20 \mathrm{~cm}$.

Frações constituintes

$\%$ dos constituintes

Areia muito grossa $(2-1 \mathrm{~mm})$

Areia grossa (1-0,5 mm)

Areia média $(0,5-0,25 \mathrm{~mm})$

29,9

Areia fina $(0,25-0,10 \mathrm{~mm})$

45,6

Areia muito fina $(0,10-0,05 \mathrm{~mm})$

5,0

Areia total

83,0

$\operatorname{Limo}(0,05-0,02 \mathrm{~mm})$

4,3

Argila $(0,002 \mathrm{~mm})$

12,7

A densidade real apresentou um valor de $2,62 \mathrm{~g} / \mathrm{cm}^{3}$, e a densidade aparente o valor de $1,35 \mathrm{~g} / \mathrm{cm}^{3}$. 


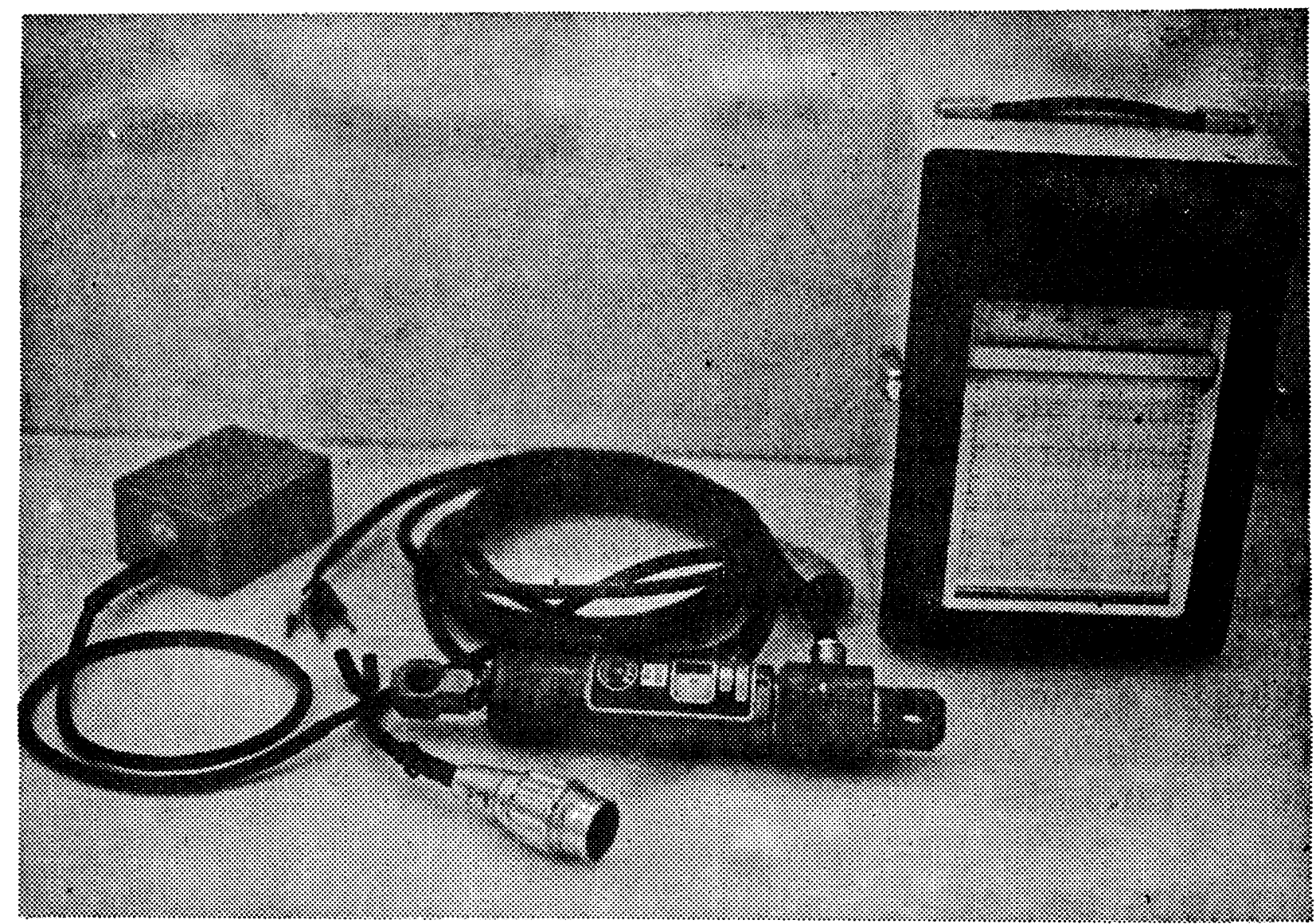

FIGURA 1 - Dinamógrafo utilizado para medição e registro dos valores da componente longitudinal do esforço tratório.

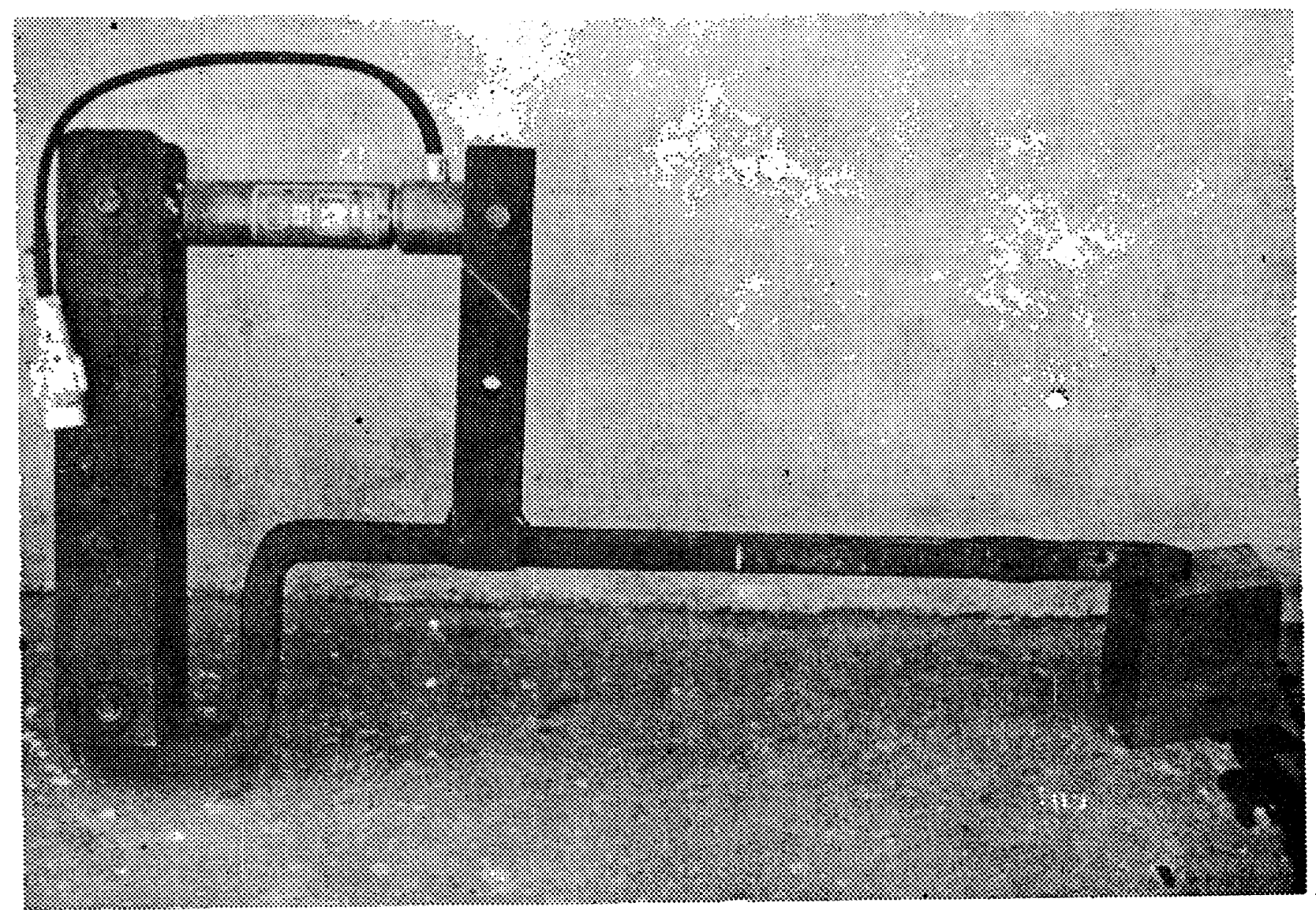

FIGURA 2 - Dispositivo de acoplamento do dinamômetro ao trator. 


\section{Métodos}

Com a finalidade de se estabelecer um melhor controle local, o delineamento experimental utilizado foi o de blocos ao acaso, com 4 tratamentos e 6 repetições. As velocidades foram designadas respectivamente em ordem crescente $V_{1}, V_{2}, V_{3}$ e $V_{4}$, correspondentes a 2. ${ }^{a}$ reduzida a 2.000 $\mathrm{rpm}$; $3 .^{\mathrm{a}}$ reduzida a $1.200 \mathrm{rpm} ; 3 .^{\mathrm{a}}$ reduzida a $2.000 \mathrm{rpm}$ e a $1 .^{\mathrm{a}}$ simples a $2.000 \mathrm{rpm}$.

A distância percorrida em cada parcela foi de 20 metros e o tempo gasto devidamente cronometrado. Foram determinados 3 perfis de secção de corte do arado em cada parcela.

0 teor de umidade do solo foi obtido em cada parcela, a partir de amostras retiradas da parede lateral do sulco de aração, logo após a passagem do arado. 0 método empregado na determinação da umidade, foi o gravimétrico padrão com secagem em estufa a $105^{\circ} \mathrm{C}$.

Os resultados obtidos foram analisados estatísticamente aplicando-se análise de variância.

\section{RESULTADOS OBTIDOS}

Os dados obtidos nos ensaios de campo, para as 4 velocidades ensaiadas, $V_{1}, V_{2}, V_{3}$ e $V_{4}$, são apresentados no QUADRO 2.

Os dados de velocidade foram obtidos, cada um de uma única observação e os demais, para umidade e áreas das secções de corte, são valores médios de três repetições dentro da parcela. 0 valor da componente longitudinal do esforço tratório é médio, calculado pela integração de área no gráfico do registrador.

QUADRO 2 - Valores dos dados obtidos nos ensaios de campo, em solo de classe textural areia, para as velocidades $V_{1}, V_{2}, V_{3}$ e $V_{4}$.

\begin{tabular}{|c|c|c|c|c|c|}
\hline Repetiços & $\begin{array}{l}\text { Velocidade } \\
\mathrm{km} / \mathrm{h}\end{array}$ & $\begin{array}{l}\text { Umidade } \\
\text { média } \\
\%\end{array}$ & $\begin{array}{l}\text { Áreas módias } \\
\text { das secçöes } \\
\text { de cort } \theta-\mathrm{dm}^{2}\end{array}$ & $\begin{array}{c}\text { Valor médio da } \\
\text { componente lon- } \\
\text { gitudinal do es- } \\
\text { forço tratório } \\
\text { kgf }\end{array}$ & $\begin{array}{l}\text { Forca especifi- } \\
\text { ca média } \\
\mathrm{kgf} / \mathrm{dm}^{2}\end{array}$ \\
\hline$v_{1}$ & 2,86 & 8,36 & 12,91 & 840 & 64,97 \\
\hline$v_{2}$ & 3,61 & 8,46 & 12,97 & 1.040 & 80,64 \\
\hline$v_{3}$ & 4,74 & 9,29 & 14,30 & 1.033 & 72.78 \\
\hline$v_{4}$ & 6,64 & 8,60 & 13,97 & 1.115 & 80,12 \\
\hline
\end{tabular}




\section{ANALISE E DISCUSSAO DOS RESULTADOS}

O efeito da variação da velocidade de deslocamento do arado sobre a força específica média foi avaliado através da análise estatística dos dados obtidos nos ensaios de campo. A análise da variância apresentada no QUADRO 3 revelou significância ao nível de $1 \%$ de probabilidade, isto autoriza a concluir-se que houve efeito do aumento da velocidade sobre a força específica média nesse tipo de solo.

QUADRO 3 - Análise da variância dos dados referentes ao efeito do aumento da velocidade de deslocamento do arado sobre a força específica média, em um solo de classe textural areia.

\begin{tabular}{lcccc}
\hline Causa de Variação & G. L. & S. Q. & Q. M. & F \\
\hline Tratamentos & 3 & 983,78 & 327,93 & $16,77++$ \\
Blocos & 5 & 383,71 & 76,74 & 3,72 \\
Resíduo & 15 & 293,31 & 19,55 & \\
\hline Total & 23 & $1.660,80$ & & \\
\hline
\end{tabular}

C. V. $=5,92 \%$

TELISCHI, MCCOLLY e ERICKSON (1956), observaram que a função $\mathrm{y}=\mathrm{f}(\mathrm{x})$, onde $\mathrm{y}$ é componente longitudinal do esforço tratório $\mathrm{x}$ a velocidade de deslocamento, tende para uma reta particular do tipo y igual a uma constante para o solo arenoso ou solo com teor de umidade baixa. Para os solos arenss e argilsos, ROWE (1959) encontrou, para a mesma função, dentro das velocidades estudadas, a variação linear $y=a x+b$, com a e b diferentes de zero.

Tendo-se em vista resultados anteriores e considerando-se a configu ração de uma série de gráficos preliminares traçados com os dados obtidos no presente trabalho, num par de eixos ortogonais com escala linear, resolveu-se admitir a função $K+f(v)$, onde $K$ é a força específica média e $v$ a velocidade de deslocamento, como sendo linear do tipo $K=m v+b$. Essa escolha atende também às dificuldades de se obter no campo uma faixa ampla de valores de velocidades que permitem a extrapolação de dados para projeto.

No QUADRO 4 estão os parâmetros referentes a ajustagem da curva de regressão, com seu respectivo coeficiente de variação. 
QUADRO 4 - Análise da variância da regressão linear para o solo de classe textural areia.

\begin{tabular}{lcrrc}
\hline Causa de Variação & G. L. & S. Q. & Q. M. & F \\
\hline Regressão.linear & 1 & 342,16 & 342,16 & $5,75^{++}$ \\
Resíduo & 22 & $1.308,21$ & 59,21 & \\
\hline Total & 23 & $1.650,37$ & & \\
\hline
\end{tabular}

C. V. $10,33 \%$

Na FIGURA 3 consta em gráfico a reta ajustada, assim como os pontos referentes as médias das repetições. A equação encontrada para o solo de classe textural areia foi:

$$
\mathrm{K}=2,64 \mathrm{v}+62,88
$$

Esta equação permite estimar-se a força específica média em $\mathrm{kgf} / \mathrm{dm}^{2}$ para um dado valor da velocidade de aração em $\mathrm{km} / \mathrm{h}$.

\section{CONCLUSOES}

Para as condições em que os ensaios foram desenvolvidos, os resultados obtidos, após uma análise e interpretação estatística, permitem chegar-se as seguintes conclusões:

A. o aumento da velocidade de deslocamento do arado provoca um consequente acréscimo na exigência da força específica média, o que, em última análise, representa um aumento na componente longitudinal do esforço tratório.

b. para o solo de classe textural areia, estabeleceu-se a função $\mathrm{K}=$ $2,64 \mathrm{v}+62,88(\mathrm{C} . \mathrm{V} .=10,33 \%)$ como a mais indicada para se avaliar a força específica média esperada em função da velocidade, sendo $\mathrm{v}$ em $\mathrm{km} / \mathrm{h}$ e $\mathrm{K}$ em $\mathrm{kgf} / \mathrm{dm}^{2}$. 


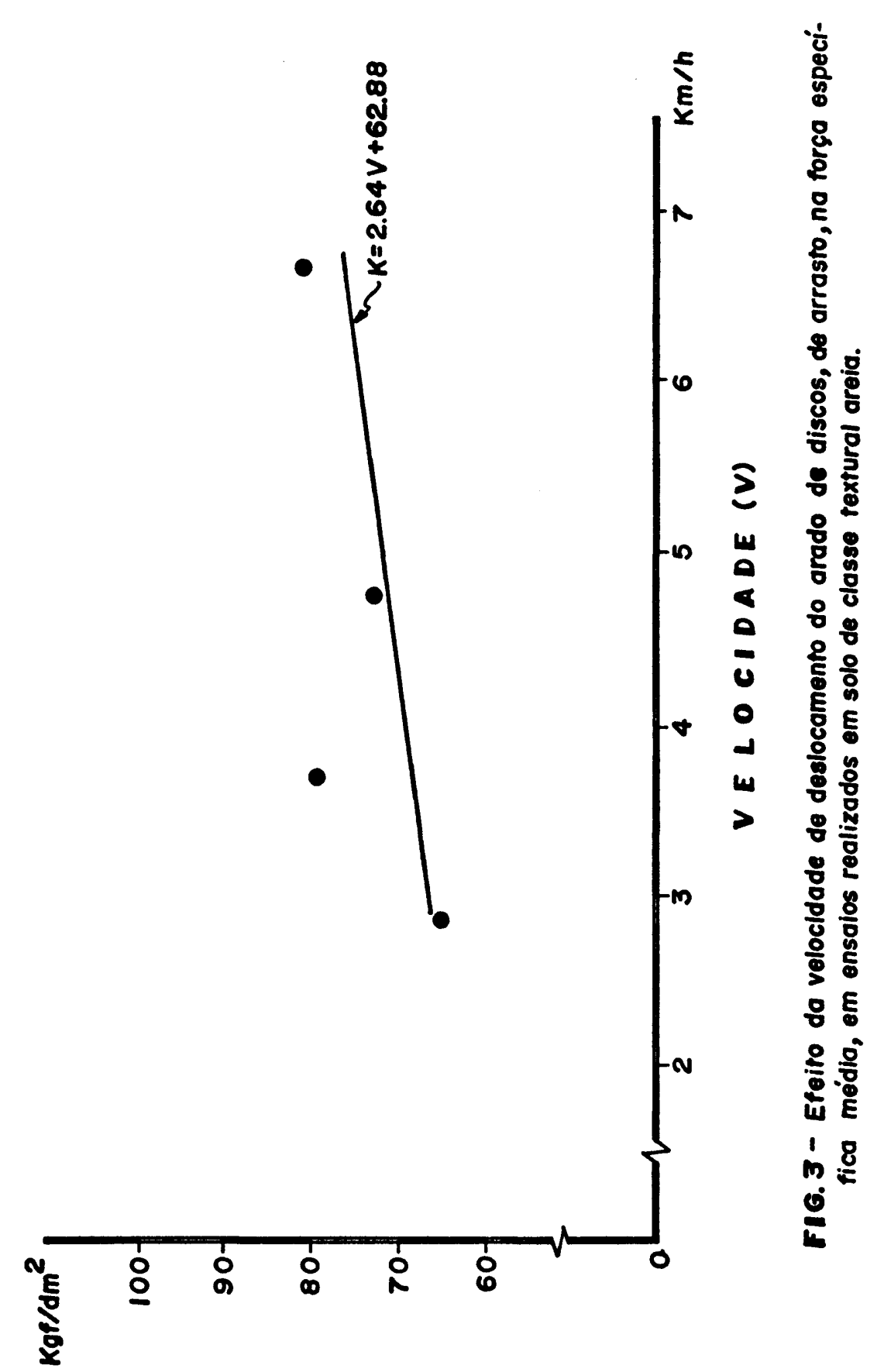

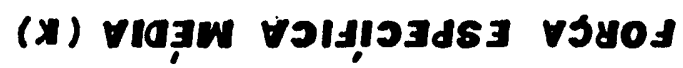

c. os valores apresentados são limitados as variações de velocidade estudadas e às condições do presente ensaio. Essas limitações, acrescidas das dificuldades em se controlar as variáveis em condições de campo, sugerem que a ampliação da faixa ensaiada, com melhor definição das rela- 
ções entre os parâmetros envolvidos, somente poderão ser obtidos em laboratório, em caixas ou tanques de solos.

d. o presente trabalho, tendo levado em consideração apenas a influência da velocidade sobre a força específica média, sem considerar o efeito da energia sobre o solo, sugere que investigações mais amplas devam ser realizadas visando avaliar o ef eito desse aumento sobre as características físicas do solo, efeito esse que tem grande importância no desenvolvimento das culturas.

\section{SUMMARY}

\section{EFFECT OF SPEED UPON THE DRAFT, ON TRAIIED DISK PLOW, IN SOIL OF SAND TEXTURAL CLASS.}

The present work was made with the purpose of studying the effect of speed increase on the values of draft. It was used a trailed disk plow. For the draft measurement it was used a coupling device in the drawbar, where it was inserted an electromechanical dynamometer PIAB.

The tests were carried on field conditions, in soil of sand textural class. Four speed were used: $2,86 \mathrm{~km} / \mathrm{h}, 3,61 \mathrm{~km} / \mathrm{h}, 4,74 \mathrm{~km} / \mathrm{h}$ and 6,64 $\mathrm{km} / \mathrm{h}$.

Under test conditions, one equation was determined: $\mathrm{K}=2,64 \mathrm{~V}+$ 62,88 , were $\mathrm{K}=$ mean specific force and $\mathrm{v}=$ speed.

\section{LITERATURA CITADA}

GETZIAFF, G. \& SOHNE, W. Forces and power requirement of freely rotating and driven plough discs on hard, dry, clayey loam. (Kräfte-und Leistungsbedarf frei drehender un angetriebeber Pflugscheiben auf hartem trocknem, tonigem Lehm). Silsoe, National Institute of Agricultural Engineering, translation 106. 1959. 22 p.

JOHNSTON, R. C. R. \& BIRTWISTLE, R. Wheatland disc plough investigations. $\Pi$. Discs forces. Journal of Agricultural Engineering Pesearch. 8(4) : 312-326. 1963.

McKIBBEN, E. G. \& REED, I. F. The influence of speed on the performance characteristics of implements. Paper presented at SAE National Tractor Meeting (Mimeo. rept.) Milwaukee, Wisconsin Sept. 1952. 5 p.

MIALHE, L. G. \& SILVEIRA, G. M. Dinamometria de arados de discos, de arrasto, em solos arenosos - ensaios preliminares. Solo. (Piracicaba, SP) 63 (1) :6770. 1971.

PAYNE, P. C. J. The relationship between the mechanical properties of soil and the performance os simple cultivation implements. Journal of Agricultural Engineering Research 1(1) : 23-50. 1956.

ROWE, R. J. Effect of speed on the elements of draft of a simple tillage tool. M.S. Thesis. Iowa State College. Ames, Iowa. 1959. $94 \mathrm{p}$.

TELISCHI, B., McCOLLY, H. F. \& ERICKSON, E. Draft measurement for tillage tools. Agricultural Engineering 37(9):605-608, 617. 1956. 